\title{
Latency and viral persistence in HIV-1 infection
}

\author{
Janet D. Siliciano and Robert F. Siliciano \\ See related article, \\ pages 839-845.
}

Department of Medicine, Johns Hopkins University School of Medicine, Baltimore, Maryland, USA

Address correspondence to: Robert F. Siliciano, Department of Medicine, Johns Hopkins University School of Medicine, 1049 Ross Building, 720 Rutland Avenue, Baltimore, Maryland 21205, USA.

Phone: (410) 955-2958; Fax: (410) 955-0964; E-mail: rsilicia@welch.jhu.edu.

HIV-1 infection can be controlled with combinations of antiretroviral drugs, an approach known as highly active antiretroviral therapy (HAART). In patients who respond well to HAART, viremia decreases to below the limits of detection, disease progression stops, and reconstitution of the immune system begins. For the first time since the beginning of the epidemic, the possibility of curing HIV-1 infection has been seriously considered (1). However, optimism regarding the eradication of HIV1 infection is tempered by the appreciation that a latent reservoir for HIV-1 exists in resting memory $\mathrm{CD} 4^{+} \mathrm{T}$ cells $(2,3)$ and persists in patients on HAART (4-6). Hence a rebound in viremia seems inevitable if therapy is stopped, a prediction borne out in recent studies. The source of this rebound virus has not been determined. An important study by David Ho and colleagues in this issue of JCI (7) suggests that the latent reservoir may be the source of this rebound virus.

The formation of the latent reservoir for HIV-1 is best understood in the context of the normal T-cell physiology. HIV-1 replicates well in activated CD $4^{+}$ $\mathrm{T}$ cells. Most of these infected lymphoblasts die quickly $\left(\mathrm{t}_{1 / 2}=1\right.$ day), due to viral cytopathic effects and/or host immune mechanisms $(8,9)$. A small fraction of infected lymphoblasts survive and revert back to a resting state by the same process that normally generates memory $\mathrm{T}$ cells. However, in this case the memory cells harbor an integrated copy of the HIV-1 genome. Although these cells do not continue to produce virus while in a resting state, they can function as a latent reservoir, capable of reinitiating virus production upon reactivation. This reservoir is extremely stable due to the fundamental biology of memory cells, which must survive for many years to provide protection against previously encountered antigens. The observed decay rate of latently infected resting $\mathrm{CD}^{+}$cells is extremely slow in most patients on HAART, with a half-life of 44 months in the typical patient (10). Based on an estimate of $10^{6}$ cells in the latent reservoir (3), 73 years of therapy would be required for eradication.

To determine whether this latent reservoir is a source of the rebound viruses that appear following interruption of HAART, Zhang et al. (7) examined variable regions of the HIV-1 envelope (env) genes of rebounding viruses in eight patients who went off of effective HAART regimens. Using a PCR assay for length polymorphisms in variable regions of env, the authors compared sequences from rebounding viruses with those from viruses isolated from resting $\mathrm{CD}^{+} \mathrm{T}$ cells during treatment. In five patients, env sequences from rebound viruses were identical in length to sequences in the latent reservoir. The authors conclude that in this subset of patients, the rebound virus was likely derived from latently infected $\mathrm{CD} 4^{+} \mathrm{T}$ cells. However, env sequences amplified from initial rebound viruses from the other three patients were different in length from latent reservoir sequences. Two of these patients also had evidence of ongoing virus production on HAART. Using a different approach, Chun et al. have also observed similarities between the rebound virus and viruses in the latent reservoir in some but not all patients (11). These authors have interpreted their results differently, focusing on the idea that HIV-1 may persist not only in the latent reservoir but in additional as-yet undefined sites as well.

\section{Caveats and questions}

Both of these studies provide insights into mechanisms of viral persistence. There are caveats, however, related to sampling and founder effects. Individual viral variants that are released from a reservoir at the time HAART is stopped will have a chance to expand unhindered by antiretroviral drugs. The rebound virus may therefore be derived from a small number of cells in the reservoir that happened to be activated when HAART was stopped. If the viral repertoire is diverse, this rebound virus represents only a small sample of the reservoir and may or may not match samples obtained directly from the reservoir depending on the degree of viral diversity and the number of samples analyzed. Therefore, observed differences between the latent reservoir virus and the rebound virus may simply result from insufficient sampling and cannot be used to say definitively that the rebound virus came from a particular location. Sampling is less of a concern in the study of Zhang et al. (7), due to the fact that the patients studied were all started on HAART within 90 days of infection, conditions that limit viral diversity. The simplest interpretation of both studies is that, in many cases, viruses in the latent reservoir resemble the rebound virus; where differences exist, they may reflect sampling problems, the presence of additional long-term reservoirs for HIV1, or the failure of HAART to completely halt viral replication.

Zhang et al. (7) go on to suggest that in the patients who have the best suppression of viral replication on HAART, the latent reservoir and rebound viruses will be genetically matched, but that in patients who have evidence of incomplete suppression, the rebound virus will derive from a population that has continued to replicate at low levels and will be distinct from the latent reservoir. Recent studies have indeed documented the inability of the HAART regimens to completely suppress virus production (12-14). Of course, continued low-level virus production could reflect release of virus from some stable reservoir without the generation of newly infected cells. However, the detection of labile replicative intermediates $(13,15)$ or temporal sequence evolution $(12,16)$ suggests that new cycles of infection may occur even in patients who have responded well to HAART. 